\title{
Reply to the letter to the editor: Development of digital shade guides for color assessment using a digital camera with ring flashes
}

\author{
Oi-Hong Tung • Yu-Lin Lai • Yi-Ching Ho • \\ I-Chiang Chou $\cdot$ Shyh-Yuan Lee
}

Received: 30 May 2013 / Accepted: 3 June 2013 / Published online: 14 July 2013

(C) Springer-Verlag Berlin Heidelberg 2013

We thank Drs. Zhuang and Zhu for their interest in our paper [1]. However, it seems that they misinterpreted our data.

Drs. Zhuang and Zhu raised a question about the color transformation method used in our study. They suggested that the camera profile should be used to transform the images RGB values into $L^{*} a^{*} b^{*}$ for color comparisons. In general, it is correct that the color information of exported pictures from a digital camera is device-dependent. To preserve more information of captured scenes in digital images, serious photographers often save these information in raw format. Raw files are a record of the unprocessed sensel data which exceed all gamut limits. We can pick the appropriate color space for each processed copy of raw files using postprocessing software to change the appearance of images on monitors to what we preferred. If we want to reproduce the images on the computer monitor as what we have seen on the camera screen after shooting, we better save the captured file in raw format and set the Adobe Photoshop with the specific camera profile. However, the intent of our research was not to duplicate the camera manufacturers' color appearance on the monitor but to show the reliability of color matching using digital images. Consequently, there is no need of strictly using the specific camera profile to read out the device's RGB values. In addition, different cameras render color in a different way and there is no perfect answer for the rendering technique. Instead of matching the camera manufacturers' color appearance, we used a spectrophotometer to measure the color parameters of the specimens as the gold standard and calibrated the images of the standardized specimens accordingly. The results of our study are promising and the technique seems to be simple and reliable for color matching using digital images.

Additionally, the comment of retrieving $L^{*} a^{*} b^{*}$ values from the camera profile seems rather impractical because most digital cameras do not directly provide $L^{*} a^{*} b^{*}$ readouts and Adobe only released limited camera profiles for some specific digital cameras.

Again, we appreciate Drs. Zhuang and Zhu for their suggestions and we hope that this reply will clean out some of their concerns.

\section{Reference}

1. Tung OH, Lai YL, Ho YC, Chou IC, Lee SY (2011) Development of digital shade guides for color assessment using a digital camera with ring flashes. Clin Oral Investig 15:49-56
O.-H. Tung $\cdot$ Y.-L. Lai $\cdot$ Y.-C. Ho $\cdot$ I.-C. Chou $\cdot$ S.-Y. Lee $(\bowtie)$ National Yang-Ming University, Taipei, Taiwan, Republic of China e-mail: sylee@ym.edu.tw 